\title{
Strategies to Increase Time in Range in Children Wearing Continuous Glucose Monitors
}

\author{
Will Bridgeman, Linda DiMeglio \\ Indiana University School of Medicine, Division of Pediatric Endocrinology
}

\section{Background and Hypothesis:}

Optimal management of Type 1 Diabetes (T1D) requires careful orchestration of insulin dosing with blood glucose values, food intake, activity levels, and concurrent illness. This is particularly burdensome for caretakers of very young children. Continuous glucose monitors (CGMs) provide useful real-time blood glucose information. Yet, data suggest that their use in young children can reduce severe hypoglycemia but does not consistently improve the time that blood glucose values are in an optimal range.

\section{Project Methods:}

We first analyzed survey data from a study of CGM use in 143 children 2-8 years of age). We then developed a semi-structured qualitative interview, and queried parents of children using CGM under 9 years of age (mean age 5.2 \pm 1.6 years, A1c $7.8 \pm 0.9 \%)$.

\section{Results:}

Recurrent themes identified included: CGMs and remote monitoring brought parents peace of mind and permitted more flexibility in care. Trend arrows indicating rapid glucose decreases induced "anxiety" and "panic" in caretakers, due to fear of hypoglycemia and immediate safety. Rising trend arrows induced frustration, but were less likely to change management decisions, due to lack of immediacy of risk of long-term complications. CGM data can be "overwhelming", but third party apps and "experience" increase helpfulness.

\section{Conclusion/Potential Impact:}

Our initial data suggest that behavioral/educational interventions to improve time in range must incorporate implementable strategies, potentially using apps, to encourage parents to respond to and reduce time spent in higher blood glucose ranges. 